\title{
Can telemedicine improve CPAP adherence?
}

\author{
Mila Kwiatkowska, ${ }^{1}$ Najib Ayas ${ }^{2}$
}

\begin{abstract}
Obstructive sleep apnoea (OSA) is a common, important disease associated with daytime sleepiness and a reduced quality of life. Moreover, untreated OSA is associated with increased healthcare utilisation and a significantly increased risk of motor vehicle crashes, hypertension, coronary artery disease and stroke. ${ }^{1}$ Treatment of patients with continuous positive airway pressure (CPAP) improves quality of life and daytime sleepiness. CPAP treatment may also substantially reduce rates of cardiac events and motor vehicle crashes. ${ }^{23}$

One of the major barriers to CPAP treatment is adherence. Many patients reject, discontinue or use it irregularly or suboptimally. The rate of CPAP discontinuation in the first 3 years of prescription ranges from $12 \%$ to $25 \%{ }^{4}$ When patients do not use CPAP sufficiently, clinical outcomes are compromised, ${ }^{5}$ demonstrating that optimising adherence is an important aspect of patient management.
\end{abstract}

\section{METHODS FOR IMPROVING CPAP ADHERENCE}

Adherence to treatment is influenced by many factors: biomedical (severity of the disorder, CPAP side effects, therapeutic response), psychological (patient's selfefficacy, anxiety, claustrophobia, the patient's perception of the seriousness of the disorder, belief that the treatment will be effective, patient's understanding of the CPAP treatment process), social (family support), technological (simplicity of the procedure, selection of CPAP device, appropriate air pressure) and economic (cost and insurance coverage). Studies have demonstrated that adherence can be significantly improved by a comprehensive support programne and timely interventions by health professionals. ${ }^{6}$

\footnotetext{
${ }^{1}$ Computing Science Department, Thompson Rivers University, Canada; ${ }^{2}$ Divisions of Respiratory and Critical Care Medicine, Department of Medicine, University of British Columbia, Canada

Correspondence to Najib Ayas, Division of Critical Care Medicine and Department of Medicine, St. Pauls Hospital, 224 Comox Building, 1080 Burrard Street, Vancouver, BC, Canada. V6Z 1Y6; najib.ayas@vch.ca
}

\section{TELEMEDICINE TO SUPPORT CPAP TREATMENT}

Telemedicine can be defined as 'the use of information and communication technology (ICT) to deliver health services, expertise and information over distance, geographic, time, social and cultural barriers'. Telemedicine encompasses various technologies-from the initial methods of store-and-forward technology of sending digital images, through the use of fax machines, e-mails, and real-time technology such as telephone, videoconferencing and systems based on the internet and web. In the last decade, telemedicine has become widespread, and applications cover the majority of the medical disciplines-from paediatrics (telepaediatrics) through radiology (teleradiology) to geriatrics (telegeratrics).

Telemedicine has been used in previous studies to promote and reinforce CPAP treatment. The results have been mixed. Taylor and colleagues randomised 114 patients to either a telemedicine (intervention) arm or traditional care. ${ }^{8}$ In the intervention arm, a cognitive behavioural intervention was applied. Questions related to CPAP use, hours of sleep and quality of sleep were sent to patients via a computer. The patients' responses were monitored by the sleep medicine practitioner, and the patient telephoned if deemed necessary. Compliance was not significantly different between the traditional and intervention groups (4.22 vs $4.29 \mathrm{~h}$ night; $\mathrm{p}=0.87$ ). The authors proposed that failure to show benefit may have resulted from difficulties in contacting patients (sometimes delayed several days). Furthermore, the intervention only provided self-reported data to the healthcare provider; objective compliance and detailed physiological information may have been more useful in effectively troubleshooting problems and may have improved CPAP compliance.

Smith and colleagues randomised 19 patients who were non-adherent with CPAP for 3 months to either a telemedicine intervention or placebo. ${ }^{9}$ Both groups were scheduled for telemedicine sessions with a nurse (14 30 min sessions over 12 weeks) using a video teleconferencing system in the patient's home. The intervention group received specific advice about CPAP whereas the placebo group had advice focused on a neutral health topic (ie, vitamins). Although the numbers of patients was small, by the end of the study, there was significantly greater rate of adherence ( $90 \%$ vs $44 \%, p=0.033$ ) in the intervention group compared to the placebo group. In a small study by DeMolles et al, a computer telephone system in addition to usual care was used in 15 patients and compared to usual care only in another 15 patients. ${ }^{10}$ The group using the telemedicine system had better CPAP adherence by the end of the study that trended to significance (4.4 vs $2.9 \mathrm{~h} / \mathrm{night}, \mathrm{p}=0.08$ ).

In this issue of Thorax (see page 1061), Sparrow and colleagues report on the use of a telemedicine intervention to improve CPAP adherence in a relatively large randomised controlled study (250 patients with sleep apnoea). ${ }^{11}$ In the intervention arm, an automated telephone-linked communication (TLC) system was tailored to promote CPAP use and designed around the concepts of motivational interviewing. Every week for the first month and every month thereafter, patients would telephone the system and report perceptions and experiences with OSA and CPAP (including compliance) over the previous week. Tailored feedback and counselling were provided by telephone to motivate patients to use CPAP. The control arm received general health information through the TLC system. At 12 months, median CPAP use was significantly greater in the intervention arm (2.98 h vs $0.99 \mathrm{~h} / \mathrm{night})$.

The study by Sparrow and colleagues suggests a potentially useful role for telemedicine in the management of patients with OSA. However, many questions remain before this specific technology or telemedicine in general can be considered for broad application. First, this study was done in only two academic medical centres, one of which was a Veterans Administration (VA) Hospital. The CPAP compliance in the control arm was relatively low, and the generalisability of the study can be questioned. Multicentre studies of telemedicine technologies in a broad range of academic and community sleep centres would be needed to address this issue. Secondly, the cost implications of this technology are unclear. Although outcomes might be improved, the incremental costs of these improved outcomes (eg, cost per additional quality-adjusted life year saved) need to be clear before policy decisions can be made. 
Thirdly, and perhaps most importantly, the telemedicine components that would be most crucial to improving adherence need to be better understood, so that the most efficient system can be designed. The current study used self-reported information and was focused on motivating patients to use CPAP. However, current technology can monitor CPAP pressures, leaks and objective compliance and send this information to the practitioner on a daily basis. Could early intervention using this information improve CPAP adherence even more? Alternatively, is it the regular automated telephone followups that are most important in motivating patients to use the device? Does the patient perception of being continuously monitored by their healthcare team (whether or not they actually are) serve as the main motivator for adherence?

Despite these caveats, we believe it likely that telemedicine will be integrated into the care of patients with OSA in the near future. The potential benefits in terms of monitoring, early intervention of problems, patient education, improving adherence and improving patient outcomes should outweigh technology costs. However, the most cost-efficient way to do this is yet to be determined.

Competing interests NA has received a research grant from Respironics Inc. MK has no competing interests.

Provenance and peer review Commissioned; not externally peer reviewed.

Published Online First 26 October 2010

Thorax 2010;65:1035-1036.

doi:10.1136/thx.2010.140897

\section{REFERENCES}

1. Al Lawati NM, Patel SR, Ayas NT. Epidemiology, risk factors, and consequences of obstructive sleep apnea and short sleep duration. Prog Cardiovasc Dis 2009;51:285-93.

2. George CF. Sleep. 5: driving and automobile crashes in patients with obstructive sleep apnoea/hypopnoea syndrome. Thorax 2004;59:804-7.

3. Marin JM, Carrizo SJ, Vicente E, et al. Long-term cardiovascular outcomes in men with obstructive sleep apnoea-hypopnoea with or without treatment with continuous positive airway pressure: an observational study. Lancet 2005:365:1046-53.
4. Engleman HM, Wild MR. Improving CPAP use by patients with the sleep apnoea/hypopnoea syndrome. Sleep Med Rev 2003;7:81-99.

5. Doherty LS, Kiely JL, Swan V, et al. Long-term effects of nasal continuous positive airway pressure therapy on cardiovascular outcomes in sleep apnea syndrome. Chest 2005;127:2076-84.

6. Zozula R, Rosen R. Compliance with continuous positive airway pressure therapy: assessing and improving treatment outcomes. Curr Opin Pulm Med 2001:7:391-8.

7. Reid J. A Telemedicine Primer: Understanding the Issues. Billings, MT: Innovative Medical Communications, 1996.

8. Taylor $\mathbf{Y}$, Eliasson A, Andrada T, et al. The role of telemedicine in CPAP compliance for patients with obstructive sleep apnea syndrome. Sleep Breath 2006; 10:132-8

9. Smith CE, Dauz ER, Clements F, et al. Telehealth services to improve nonadherence: a placebocontrolled study. Telemed J E Health 2006;12:289-96.

10. DeMolles DA, Sparrow D, Gottlieb DJ, et al. A pilot trial of a telecommunications system in sleep apnea management. Medical Care 2004:42:764-9.

11. Sparrow D, Aloia M, DeMolles DA, et al. A telemedicine intervention to improve adherence to continuous positive airway pressure: a randomised controlled trial. Thorax 2010;65:1061-6.

\title{
BCG vaccination: 90 years on and still so much to learn ...
}

\author{
Ajit Lalvani, Saranya Sridhar
}

The history of vaccination against tuberculosis abounds with instances of scientific discovery and rediscovery. At the end of the 19th and beginning of the 20th century, following Robert Koch's announcement of his discovery of $\mathrm{Myco}$ bacterium tuberculosis in 1882, scientists across the world, including Koch himself, set out to invent a vaccine against tuberculosis. In 1908 Leon Calmette, a bacteriologist, and Camille Guérin, a veterinarian at the Pasteur Institute, Lille began an experiment to devise a vaccine by attenuating a Mycobacterium bovis strain until it lost its virulence. Thirteen years and 230 passages later, they were able to show that

Tuberculosis Research Unit, Department of Respiratory Medicine, National Heart and Lung Institute, Imperial College London, London, UK

Correspondence to Ajit Lalvani, Tuberculosis Research Unit, Department of Respiratory Medicine, National Heart and Lung Institute, Imperial College London, Norfolk Place, London W2 1PG, UK;

a.lalvani@imperial.ac.uk the strain was protective in animal models and no longer caused disease which was subsequently discovered to be primarily due to the loss of the genes in the region of difference 1 (RD1) region of the $M$ bovis genome. ${ }^{1}$ That same year, an infant tuberculosis contact was given the first dose of this live attenuated vaccine, $M$ bovis Bacille Calmette-Geurin (BCG). Now, with over 3 billion doses administered, BCG is the most widely used vaccine worldwide.

Since its first use 90 years ago, BCG has been recommended as a vaccine because of its partial protective effect against active tuberculosis and death, albeit with greater efficacy against disseminated and meningeal disease in children than pulmonary disease in adolescents and adults. ${ }^{2} 3$ This, taken together with autopsy studies suggesting BCG decreases the size of pulmonary tuberculous foci thereby limiting bacillary multiplication and spread $^{4}$ and animal models where BCG vaccination reduces bacillary burden after
$M$ tuberculosis challenge but does not protect against infection, led to the longstanding dogma that BCG protects against dissemination and disease but not against infection. It was only 5 years ago that the first report ${ }^{5}$ of the ability of BCG to protect against the acquisition of infection changed our thinking about how BCG acts. The finding that BCG can act at an early stage in the pathway from tuberculosis exposure to disease (figure 1) has since been corroborated in community-based contact investigations in adults and children in Hamburg ${ }^{6}$ and in two studies investigating school tuberculosis outbreaks in the UK, ${ }^{7}$ the latest of which is published in the current issue of Thorax (see page 1067). ${ }^{8}$ Investigating a pointsource tuberculosis outbreak at a nursery school, Eriksen and colleagues found that BCG-vaccinated children were significantly less likely to be infected, as judged by interferon-gamma release-assay (IGRA) results, than unvaccinated children. Vaccine effectiveness against infection was estimated at $66 \%$ but, given the small size of the study, the CIs are sufficiently wide to overlap with the $38 \%{ }^{7}$ and $24 \%^{5}$ reduction in RR observed in the previous larger studies. Collectively, these data strongly suggest that at least part of the protective effect of BCG is attributable to protection against infection, which has substantial 